\title{
Análisis del nivel de inclusión y posibles barreras de las técnicas de desarrollo de la inteligencia emocional y en particular del mindfulness en el área de Educación física en la ESO \\ Analysis of the inclusion level and possible barriers of the development techniques of emotional intelligence and in particular that of mindfulness in the area of Physical Education in ESO
}

\author{
Sandra Constantino M urillo, JoséAntonio Navia Manzano \\ Universidad Politécnica de M adrid (España)
}

\begin{abstract}
Resumen. El objetivo fue analizar el nivel de penetración detécnicas de desarrollo delainteligenciaemocional (IE) y en particular del mindfulness, así como las posiblesdificultades de la prácticaen laclasede educación física(EF) percibidaspor losdocentes. Seelaboró un cuestionario tipo Likert con 20 ítems con cuatro dimensiones referidosalos docentes: formación universitaria, experiencia personal, desarrollo profesional y dificultadesde aplicación en lasclases y fuedistribuido por internet al territorio Español siendo respondidapor 79 docentes de EF de la etapa de Secundaria (41 mujeres y 38 hombres), con una media de edad de $38.5 \pm 8.8$. Para determinar la fiabilidad del cuestionario se realizó el análisis exploratorio y confirmatorio, se encontró otro model o alternativo decuestionario que permitemásestudioscon mayor índice de ajusteen los resultados. Lametodologíaaplicadafue cuantitativa con un diseño transversal y procedimiento selectivo. Los resultados reflejaron queel $81 \%$ de los docentes recibieron unaescasafor mación universitariafrenteaun 19\% que afirmaron que sí la habían recibido. También se destacó el escaso desarrollo profesional (47\% planificaban contenidos para desarrollar laIE, frente a un 53\%), siendo ambos factores los que más dificultades generaron al incluir las técnicas de desarrollo de habilidades emocionales en las clases. Además se obser vó un efecto significativo en la edad, el sexo y los años de experiencia de los docentes (pd» .05) en lainclusión detécnicas de desarrollo de laIE. Los resultados concluyeron quelas dificultades más destacadas por los profesores son laescasaformación recibidaen launiversidad y el desconocimiento del mindfulness como técnicainnovadoray sus beneficios.
\end{abstract}

Palabrasclave: Educación, competenciasemocionales, beneficios, formación y habilidades.

\begin{abstract}
The objective was to analyze the level of inclusion of techniques for the development of emotional intelligence (EI) and mindfulnessin particular, as well asthe possible difficultiesperceived by theteachers when imparting a physical education $(\mathrm{PE})$ class. A Likert-type questionnaire of 20 items spread amongst four dimensions (university education, personal experience, professional development, and difficulties in applying it in class) was developed and distributed onlinew ithin the Spanish territory. $79 \mathrm{PE}$ teachers of the secondary school answered the questionnaire ( 41 women and $38 \mathrm{men}$ ), with an average of $38.5 \pm 8.8$ years. To determine the reliability of the questionnaire, an exploratory and confirmatory analysiswascarried out, bringing as result an alternative questionnaire model which provided ahigher reliability index of adjustment in the results. The applied methodology was quantitative with acrosssectional design and selective procedure. The results reflected that $81 \%$ of teacher sreceived little univer sity training compar ed to $19 \%$ who stated that they had received it, the scarce professional development was al so highlighted ( $47 \%$ planned content to devel op EI, compared to 53\%), both factors representing the main source of difficulties generation for teachers when including emotional skills development techniques in their classes. In addition, asignificant impact wasobser ved in the age, gender and years of experience of the teachers (pd» .05) including El development techniques. The results concluded that the most notabledifficultiesfor theteachersare: the scarce training received at the university and theignorance of mindfulness as an innovativetechnique and its benefits.
\end{abstract}

Keywords Education, emotional competencies, benefits, training and skills.

\section{Introducción}

Relevancia del desarrollo de la IE en el docente Se realiza una aproximación significativa a los docentes como principal fuente de conocimiento y formación que los alumnos reciben diariamente, enfatizando la importancia que su capacidad y destreza en las habili-

Fecha recepción: 20-10-20. Fecha de aceptación: 29-01-21

Sandra Constantino Murillo

sandrix 1979@ hotmail.com dades en IE y su inclusión en la enseñanza son necesarias en el aula (Álvarez, Bisquerra, Fita, Martínez \& Pérez, 2000; Berrocal \& Extremera, 2002). Viene relacionado y suscitado con la definición de IE como «La habilidad para percibir, val orar y expresar emociones con exactitud, la habilidad para acceder $\mathrm{y} / \mathrm{o}$ generar sentimientos quefaciliten el pensamiento; la habilidad para comprender emociones y el conocimiento emocional y la habilidad para regular las emociones promoviendo un crecimiento emocional e intelectual» (Mayer \& Salovey, 1997), Berrocal \& Extremera (2002) afirman que esta 
habilidad amplía la capacidad de percibir, asimilar y comprender los sentimientos de una manera adecuada, la facultad de comprenderlos y la agilidad para normalizar el propio estado anímico y el de los demás.

\section{La IE en los ámbitos Educativos y D eportivos}

Según Berrocal \& Ruiz (2008), con el siglo XXI en Españase ha implantado unamayor conscienciaen cuanto a la importancia de ofrecer una educación social y emocional en la escuela, persiguiendo la resolución de conflictos en el colectivo educativo, ofreciendo las herra mientas que más se ajusten tanto al desarrollo de las habilidades emocionales como a las personales. Espada $\&$ Calero (2012), consideran la asignatura de EF como una vía excelente para trabajar estos aspectos que ayudan a desarrollar la IE. Es preciso programar y llevar a cabo sesiones emprendedoras y dinámicas de tareas individuales y grupales, de esta forma, los alumnos podrán experimentar e interiorizar la IE con el objetivo de que aprendan a reconocer, controlar y gestionar las propias emociones y desarrollar sus destrezas sociales.

Fernández \& Navarro (1989) afirman que los aspectos físico, motriz, afectivo y social, son términos esenciales que constituyen el soporte de la EF, incluyendo posteriormente los aspectos afectivo y social en el currículum, debido a la gran importancia y relación con la IE. El equipo de investigación de Mañas et al. (2014) resalta que es preciso que los docentes tengan la labor de fomentar una práctica de diferentes técnicas (e.g, mindfulness) para que se desarrollen las competencias emocionales en el aula «Los docentes han de asumir responsabilidades que conlleven ejercitar la consciencia propia y autoconocimiento, la percepción y regulación de las propias emociones, la tolerancia, las habilidades interpersonales e intrapersonales, el afecto hacia los demás y hacia uno mismo, el respeto y la atención» (p. 195). Constantino \& Espada (2021) plantea en su investigación una unidad didáctica basada en mindfulness y biodanza para desarrollar la IE, afirman que el empleo de estas técnicas favorece el desarrollo de la IE en cuanto a la atención emocional, claridad emocional y repa ración de las emociones.

Sobre la IE en el deporte se proyectaron métodos de desarrollo de la IE en la planificación de los deportistas con el fin de reducir y dominar el estrés (Riera, 1985). Investigaciones más recientes como la deAcebes \& Rodríguez (2019) plantean que «Las actividades físico-deportivas presentan el contexto idóneo para plantear la percepción y expresión de emociones, comprensión emocional o recrean situaciones de una necesaria regulación emocional» (p.300).

\section{El Mindfulness y contexto escolar}

Bisquerra (2008) propone una serie de condiciones a tener en cuenta por los docentes para introducir la IE en el ámbito educativo, donde primeramente se proyecta un soporte y fundamentación conceptual sobre el mindfulness para llevarlo a la práctica y la formación adecuada.

El pionero Kabat-Zinn (2010), menciona que el mindfulness es «Prestar atención de una manera especial: intencionadamente, en el momento presente y sin juzgar» (p. 18), hace referencia al desarrollo de las habilidades que consiguen una mejora en la atención de lo que se vive en cada momento. Desarrolla el programa MBSR (M indfulness-Based Stress Reduction), con ocho semanas de duración en la Universidad de M assachusetts para pacientes con dolor crónico. Con sorprendentes resultado, fue promovido al ámbito educativo. Actualmente se sigue practicando por los numerosos beneficios que conlleva su práctica. Según Cardaciotto (2005), el mindfulness engloba dos componentes diferenciados: la observación en el instante presente, que efectúa una continua búsqueda de las prácticas externas designadas a los eventos contextuales, como a las internas (pensamientos, emociones, etc.) y la aceptación o comprensión de lo que nos sucede sin una predisposición defensiva (indispensable para avanzar y poder realizar cambios reales). Para concluir, González (2013) recoge y aclara en su guía para educadores que «El mindful ness no consiste en relajarse, no es renunciar a nuestras emociones, no tiene que ver con apartarnos de la vida, no es difícil de practicar, únicamente requiere una rutina, no es convertirnos en una nueva religión, no se trata de transcender la vida ordinaria, no representa mantener la mente en blanco o de desocuparla de pensamientos y no es rehuir del dolor».

\section{Programas de Mindfulness en los ámbitos Edu- cativos y Deportivos}

En los 90 la educación comenzó a ser más organizada y estructurada, incorporando el mindfulness en diferentes etapas. LaAsociación «M indful Schools» (2000), emergió en California y sigue en auge mundialmente reconocida con el mismo nombre: Mindful Schools (2007). El programa de entrenamiento de mindfulness emergía con un periodo de cinco semanas, desarrollando de tres a cuatro sesiones con 15 minutos casa sesión. Los resultados fueron una mejora a corto plazo por parte de los docentes de primaria y que los alumnos dismi- 
nuyeron la facultad de estrés y de estar en alarma. Es por tanto de vital relevancia, mencionar los resultados obtenidos a través de las prácticas de meditación ya que se relaciona los resultados académicos y las habilidades cognitivas, viéndose favorecido las habilidades de atención y procesamiento de la información de forma más rápida y eficaz, siendo un camino fiable para su práctica (Shapiro, Warren \& Astin, 2008).

Respecto a los programas de entrenamiento en mindfulness para docentes, se destacan los llevados a cabo en Norteamérica. El programa de MindfulnessBased Wellness Education (M BWE), se creó en Toronto en el año 2015 y se le incluye al docente desde el inicio del curso. La duración es de nueve semanas y se trabaja estrategias de enseñanza con distintas dimensiones como la escucha consciente y la paz social, asignados a todos los miembros de la comunidad educativa. Múltiples investigaciones sobre el programa $M B W E$, afirman que los docentes favorecen sus niveles de autoeficacia para enseñar a los alumnos y mejoran la salud física de estos (Poulín, 2009). El programa Stress Management and Relaxation Techniques (SM ART), actual mente vigente en Vancouver y Colorado, está basado en el entrenamiento de tres aspectos principales como desencadenantes paralabuena práctica: atención, concentración y la práctica del mindfulness; la consciencia y entendimiento de las emociones y la compasión y práctica en empatía. La duración es de once sesiones. Los resultados confirman que la interacción con los al umnos y compañeros influye positivamente, al igual que la motivación en el trabajo (Ragoonaden, 2016).

En España, el mindfulness se instauró de forma sistematizada a partir del año 2012 y fue respal dada por organizaciones en el ámbito educativo. Destacar el tra bajo de Franco et al. $(2009,2010)$ en la que afirmaron que la meditación disminuía valores de ansiedad, depresión y estrés del profesorado. La aportaciones de Delgado (2009), expone datos experimentales para alcanzar una mayor calidad de vida en la carrera profesional del docente y lo propone como programa de prevención. León (2008), por su parte, afirma la estrecha relación entre el incremento de los resultados y la atención plena en estudiantes.

López, Amutio, Herrero \& Bisquerra (2016) lleva ron a cabo el ProgramaTREVA. López (2013) experto en mindfulness, relajación y espiritualidad, organizó este programa siendo el resultado de un estudio que parte de la investigación-acción (IA) y la Lesson Study (LS), considerados dos planteamientos cual itativos muy provechoso. Se desarrolló a través de un estudio cuasi-ex- perimental para adaptar el mindfulness a las necesida des de los docentes y los alumnos. Los procesos real iza dos fueron, definir el problema, diseñar el plan, desarrollar la LS y recolección de datos y su posterior reflexión. Los contenidos del programaque agrupan López et al. (2016) son nueve Lesson Study (LS), LS básicas: «atención, respiración, relajación, visualización, conciencia sensorial voz-habla, postura, energía y movimiento y tres LS especiales: mindfulness, gestión emocionalfocusing y centramiento» (p.78).

\section{Beneficios del Mindfulness e investigaciones empíricas}

En base a las anteriores aportaciones, se han ido incorporando al cómputo científico en los laboratorios las diversas maneras de meditación, estudiando la compa sión, la consciencia plena, y la atención focalizada. Existen numerosos estudios que soportan los beneficios a nivel anatómico y neurofisiológico (Luders, Clark, Narr1 \&Toga, 2011; Hölzel, 2011; Dickenson, Berkman, Arch \& Lieberman, 2013; M atthieu et al., 2015) y psicológico, entre ellos se destaca la minoración de la percepción y nivel del dolor (lasmanifestacionesfísicas en cuanto al dolor disminuyen), se observa un equilibrio emocional y mental, un aumento en cuanto a la habilidad para relajarse y autorregularse, afrontar las situaciones de estrés, proporcionando una mayor calma, estabiliza los cambios posibles de personalidad, aumenta la autoestima, promueve el autoconcepto y produce una disminución en los estados depresivos aumentando la percepción de energía (Kabat-Zinn, 1990). Favorece la capacidad y tareas atencionales, la impulsividad, desa rrollando la capacidad de percibir y manejar las situa ciones de estrés (M añas, 2014). «Suaviza todo tipo de estados de tensión, estrés, ansiedad o dolor» (Canales $\&$ Rovira, 2017, p.120). Siegel (2007) por su parte, afirma que favorece la integración neuronal, zona en la que se regula las relaciones sociales y las emociones. En el deporte (Trujillo \& Reyes, 2019) se ha demostrado la reducción de niveles de ansiedad cognitiva y somática e incremento en la autoconfianza. Por consiguiente, es necesario que los docentes tomen consciencia de que el buen acrecentamiento del dominio de las técnicas para el desarrollo de las habilidades emocionales como es el mindfulness contribuye a un destacable bienestar propio y colectivo del alumnado.

En referencia a lo anterior, el objetivo de este estudio fue analizar en los docentes de secundaria el nivel de inclusión de las técnicas en habilidades emocionales como el mindfulness, y las posibles dificultades de la 
práctica en clase de EF.

\section{M etodología}

\section{Participantes}

Participaron 80 docentes de EF de las etapas de ESO a nivel nacional, con una media de edad de $38.5 \pm 8.8$. Un participante no rellenó todos los datos necesarios, por lo que la muestra final fue de 79 participantes (41 mujeres y 38 hombres). Para la participación de los docentesse solicitó del consentimiento informado, dónde se explicaba en qué consistía el cuestionario. La distribución de las encuestas se realizó a través del correo electrónico a los docentes del INEF, a los docentes del ICE (Instituto de Ciencias de la Educación) y a los diferentes Colegios 0 ficiales de Licenciados de todas las Comunidades Autónomas para facilitar su distribución y la realización de la misma.

La investigación respetó los postulados de la Decla ración de Helsinki de 2013 y recibió la aprobación de la Comisión de Ética de la Universidad Politécnica de Madrid.

\section{Material y procedimiento}

Las percepciones de los profesores se obtuvieron a través de un cuestionario. Tras una exhaustiva búsqueda, no se encontraron cuestionarios validados de referencia que nos hubiesen podido ayudar, por lo que se elaboró un cuestionario ad-hoc. Para tal fin, se combinaron dos métodos: la búsqueda bibliográfica y la opinión experta de los profesionales del mindfulness. Se consultó a tres profesores de mindfulness sobre su opinión de estas técnicas en la ESO y los motivos que podrían favorecer la penetración (o las dificultades). Se elaboró el cuestiona rio entorno a las siguientes dimensiones a) datos personales y demográficos ( 7 ítems); b) formación en la universidad ( 5 ítems); c) experiencia personal ( 6 ítems); d) desarrollo profesional (5 ítems);e) dificultades de aplicación (4 ítems). El cuestionario (aparte de los da tos demográficos) consta de 20 ítems. Estos ítems se presentan en un formato tipo Likert de 4 puntos ( $1=$ totalmente en desacuerdo, $4=$ totalmente de acuerdo). Además, se preguntaron de forma abierta por el número de horas de práctica de las técnicas de desarrollo personal y emocional, y del mindfulness. Nótese que puntuaciones altas en la última dimensión (barreras) reflejan mayores dificultades. Para facilitar la divulga ción, se realizó la encuesta a través de la página online (https:/ / www. onlineencuesta.com). Para ejecutar el análisis y tratamiento de los datos, se extrajeron los datos directamente de la página web mediante una exportación en archivo xls, utilizando el programa Microsoft Excel 2017.

\section{Diseño y análisis}

Se empleó una metodología cuantitativa, diseño transversal, descriptivo y no experimental. Las variables dependientes fueron las puntuaciones obtenidas en el cuestionario. Dado que el tipo de variables son ordinales y que las pruebas de normalidad K-S resultaron significativas paralas cuantitativas, se llevaron a cabo pruebas no paramétricas para el análisis de los datos. En primer lugar, se realizó un análisis descriptivo de los datos demográficos y de las respuestas del cuestionario. Después, se realizaron pruebas de correlación de Rho de Spearman entre las diferentes variables y las horas de práctica de las habilidades emocionales y de las sesiones de mindfulness. Por último, se utilizó el test $U$ $M$ ann-W hitney para muestras independientes para ana lizar las diferencias por sexo y el test de K ruskall-Wallis para anal izar las diferencias por plan de estudios. El tamaño del efecto se expresó mediante la d de Cohen (1988), con valores de $0.2,0.5$ y 0.8 para tamaños pequeños, medianos y grandes, respectivamente. Los aná lisis se realizaron con los programas Jamovi V. 0.9.5.16 y SPSS versión 25.0, considerando un nivel de á $=0.05$ para todos los análisis.

\section{Resultados}

En laTabla 1, se observa que la mayoría de los participantes realizaban sus labores de docente en la Comunidad Autónoma de Madrid (56\%), seguido de Navarra (15\%), Andalucía (10\%), Valencia (6\%), Castilla-La Mancha (5\%), Extremadura y Castilla-León (3\%) y Cataluña y Canarias (1\%). Casi la mitad de los profesores estudiaron en la U niversidad Politécnica de Madrid (UPM) en el Instituto Nacional de Educación Física (INEF). En nexo a los años desempeñados como docentes de EF, el $59 \%$ afirmó haber ejercido entre 0 y 10

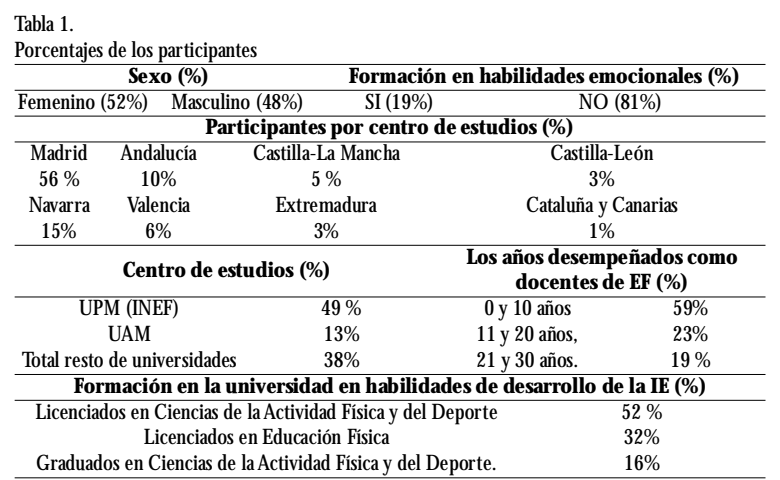


Tabla 2.

Estadísticos descriptivos de las variables dimensiones y frecuencia de losítems.

\section{Variables. Ítems}

\section{Estadísticos}

Frecuencia en \%

relativo

Dimensión formación

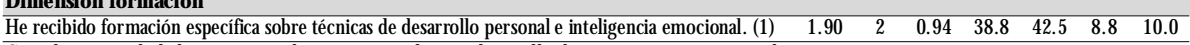

Considero que sali de la carrera, totalmente preparado para desar rollar las competencias emocionales $\begin{array}{llllllll}17.4 & 2 & 0.67 & 36.3 & 56.3 & 5.0 & 2.5\end{array}$

en mi futura labor como docente. (2)

Los profesores que tuve en la Universidad, empleaban una metodología innovadora para desar rollar

las habilidades en inteligencia emocional en sus clases, de esta forma pude adquirir técnicas de forma $\begin{array}{llllllll}1.74 & 2 & 0.69 & 37.5 & 53.8 & 6.3 & 2.5\end{array}$

indirecta.(3)

\begin{tabular}{llllllll}
\hline Los profesores prestaban más atención al desar rollo de las competencias emocionales inter personales & 1.59 & 2 & 0.61 & 46.3 & 50.0 & 2.5 & 1.3
\end{tabular}

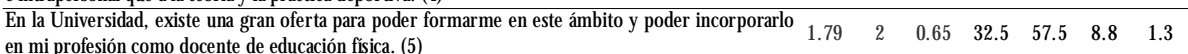

Dimensión experiencia personal

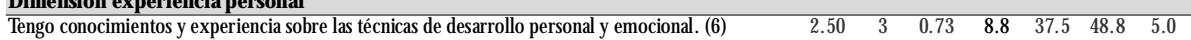

Mi disposición hacia la práctica de técnicas de desar rollo per sonal y emocional es positiva. (7) $\quad$\begin{tabular}{lllllllll}
\hline & 3.27 & 3 & 0.56 & 1.3 & 1.3 & 66.3 & 31.3 \\
\hline
\end{tabular}

$\begin{aligned} & \text { Practico técnicas de desarrollo personal y emocional semanalmente, me proporciona bienestar y me } \\ & \text { ayuda a autor regular mis estados emocionales. (8) }\end{aligned}$
2.46

\begin{tabular}{lllllllll}
\hline Tuve una experiencia negativa hacia el aprendizaje de técnicas de desar rollo per sonal y emocional, & 1.61 & 1 & 0.72 & 51.2 & 33.5 & 10.0 & 1.3
\end{tabular}

\begin{tabular}{llllllll}
\hline Conozco los principios básicos de la técnica del Mindfulness. (10) & 2.34 & 2 & 0.92 & 21.3 & 37.5 & 27.5 & 13.8
\end{tabular}

\begin{tabular}{llllllll}
\hline Conozco los principios básicos de la técnica del Mindfulness. (10) & 2.34 & 2 & 0.92 & 21.3 & 37.5 & 27.5 & 13.8 \\
\hline Practico el Mindful ness como técnica de desarrollo de las habilidades per sonales y emocionales. (11) & 200 & 2 & 0.94 & 35.0 & 36.3 & 22.5 & 6.3
\end{tabular}

\begin{tabular}{l} 
Practico el M indful ness como técnica de desarrollo de las habilidades per sonal es y emocionales. (11) \\
\hline Dimensión desarrollo profesional
\end{tabular}

En clase de EF planifico y llevo a cabo contenidos para desarrollar las habilidades emocional es y el

desar rollo personal. (12)

$47.5 \quad 52.5$

\begin{tabular}{llll}
\hline En caso afirmativo, indique el número de sesiones al año. $\left.{ }^{*}\right)$ & 4.24 & 0 & 5.97 \\
\hline
\end{tabular}

La motivación y disposición de los alumnos hacia las técnicas de desar rollo de habilidades emocionales $\quad 81.4 \quad 18.6$

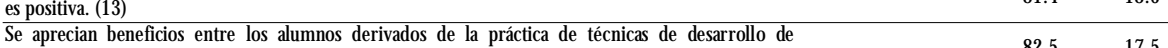

\begin{tabular}{lll} 
habilidades emocionales (14) & 82.5 & 17.5 \\
\hline Aplico la práctica del M indfulness en las clases de EF. (15) & 47.6 & 52.4
\end{tabular}

\begin{tabular}{lllll}
\hline Aplico la práctica del Mindfulness en las clases de EF. (15) & & 47.6 & 52.4 \\
\hline En caso afirmativo, indique el número de sesiones al año(*) & 1.96 & 0 & 4.82 &
\end{tabular}

\begin{tabular}{lll}
\hline Se aprecian los beneficios del mindfulness. (16) & 69.2 & 39.8
\end{tabular}

Dimensión dificultades de aplicación

\begin{tabular}{lllllllll}
\hline Mi disposición para impartir técnicas de desarrollo personal con el mindfulness en clase de EF es & 1.84 & 2 & 0.70 & 33.8 & 51.2 & 12.5 & 2.5
\end{tabular}

negativa. (17)

No practico técnicas de desarrollo per sonal en clase de EF debido a la mala disposición de los alumnos $\begin{array}{lllllll}2.26 & 2 & 0.88 & 30.0 & 53.8 & 15.0 & 1.3\end{array}$

(falta de interés, apatía, etc.). (18)

\begin{tabular}{llllllll}
\hline No practico técnicas de desarrollo personal en clase de EF debido a la falta de tiempo para 2.23 & 2 & 0.90 & 21.3 & 38.8 & 32.5 & 7.2
\end{tabular}

temporalizar y desarrollar este tipo de contenido. (19)

No practico técnicas de desarrollo per sonal en clase de EF debido a que no dispongo de los materiales

e instalaciones (equipo de música, colchonetas, tatami, acústica, temperatura de la instalación $1.75 \quad 1.80 \quad 0.57 \quad 23.8 \quad 37.5 \quad 31.3 \quad 7.5$

inadecuada, etc.). (20)

Fuente: elaboración propia Nota: M (media), Mdn (mediana), DT (desviación típica), dimensión formación (1-5), dimensión experiencia per sonal (6-

11), dimensión desar rollo profesional (12-16; *) y dimensión dificultades (17-20); completamente de acuerdo, (2) de acuerdo, (3) en desacuerdo, (4) completamente en desacuerdo.

años, consecutivo de un $23 \%$ entre 11 y 20 años, y un $19 \%$ entre 21 y 30 años. Por plan de estudios, el $52 \%$ de los participantes eran Licenciados en Ciencias de laActividad Física y del Deporte, un $32 \%$ eran Licenciados en Educación Física, y un $16 \%$ eran Graduados en Ciencias de laActividad Física y del Deporte. En cuanto a la formación en la universidad en habilidades de desarrollo de la IE, un $81 \%$ de los participantes, afirmaron que no había recibido formación frente a un $19 \%$ que sí habían recibido. Los resultados referidosal porcentaje según centro de estudios, destacó la Comunidad Autónoma de Madrid y mostraron que un $49 \%$ de la muestra estudió en laFacultad de Ciencias de laActividad Física y del Deporte (Antiguo INEF), seguido del $13 \%$ que estudió en la U niversidad Autónoma de Madrid.

en mi profesión como docente de educación física. (5)
Tabla 3.

Correlación de Rho de Spearman (?) entre las diferentes variables y las horas de práctica de las habilidades emocionales y de las sesiones de mindfulness.

Variables. Dimensiones

Sesiones de Sesiones de habilidades mindfulness

Dimensión formación

He recibido for mación específica sobre técnicas de desar rollo per sonal e inteligencia emocional.(1)

Considero que salí de la carrera, totalmente preparado para desarrollar las competencias

emocionales en mi futura labor como docente. (2)

Los profesores que tuve en la Universidad, empleaban una metodología innovadora para desarrollar

las habilidades en inteligencia emocional en sus clases, de esta forma pude adquirir técnicas de forma indirecta. (3)

Los profesores prestaban más atención al desar rollo de las competencias emocionales

interpersonales e intraper sonal que a la teoría y la práctica deportiva. (4)

En la Univer sidad, existe una gran oferta para poder formar me en este ámbito y poder incorporar 10

Dimensión experiencia personal

Tengo conocimientos y experiencia sobre las técnicas de desar rollo per sonal y emocional. (6)

Mi disposición hacia la práctica de técnicas de desarrollo per sonal y emocional es positiva. (7)

Practico técnicas de desarrollo personal y emocional semanalmente, me proporciona bienestar y me ayuda a autor regular mis estados emocionales. (8)

Tuve una experiencia negativa hacia el aprendizaje de técnicas de desar rollo personal y emocional,

bien por experiencias pasadas, falta de interés o de motivación. (9)

Conozco los principios básicos de la técnica del Mindfulness. (10)

\begin{tabular}{lll} 
Practico el Mindfulness como técnica de desar rollo de las habilidades personale & $0.28^{*}$ & $0.53^{* * *}$ \\
\hline
\end{tabular}

Dimensión dificultades de aplicación

Mi disposición para impartir técnicas de desarrollo persona como el mindfulness en clase de EF es

negativa. (17)

No practico técnicas de desarrollo personal en clase de EF debido a la mala disposición de los

alumnos (falta de interés, apatía, etc. ). (18)

No practico técnicas de desarrollo personal en clase de EF debido a la falta de tiempo para

temporalizar y desar rollar este tipo de contenido. (19).

No practico técnicas de desarrollo personal en clase de EF debido a que no dispongo de los

materiales e instalaciones (equipo de música, colchonetas, tatami, acústica, temperatura de la

instal ación inadecuada, etc.). (20).
La Tabla 2 muestra el análisisdescriptivo de losda Semográficos y de las spuestas del cuestionario, observó el escaso desa ollo profesional como los actores que más dificulta generaron al incluir las cnicas de desarrollo de El las clases (47\% afirman que si planificaban condos para desarrollar la y un $53 \%$ no planifican). Se realizó un análisis Ce (Tabla 3), con el bles relaciones entre los ferentes ítems y las sesios de habilidades emocioales y de mindfulness, se bservó una correlación uy positiva entre forma y y las sesiones en habiemocionales $(\tilde{n}=$ ; $p<.01$ ). 
Los profesores prestaban más atención al desar rollo de las competencias emocionales inter per sonales $1.51 \quad 0.551 .68 \quad 0.66 \quad 678.50 \quad 0.265 \quad-0.28$ e intraper sonal que a la teoría y la práctica deportiva. (4)

En la Universidad, existe una gran oferta para poder formar me en este ámbito y poder incor porarlo $\begin{array}{llllllll}1.73 & 0.59 & 1.87 & 0.70 & 710.00 & 0.444 & -0.21\end{array}$ en mi profesión como docente de educación física. (5)

Dimensión experiencia personal

Tengo conocimientos y experiencia sobre las técnicas de desar rollo per sonal y emocional. (6) $\quad \begin{array}{lllllllll} & 2.68 & 0.69 & 2.34 & 0.71 & 607.00 & 0.064 & 0.49\end{array}$

Mi disposición hacia la práctica de técnicas de desarrollo per sonal y emocional es positiva. (7) $\quad$\begin{tabular}{lllllllll}
3.41 & 0.50 & 3.11 & 0.56 & 575.50 & 0.015 & 0.59 \\
\hline
\end{tabular}

Practico técnicas de desar rollo per sonal y emocional semanalmente, me proporciona bienestar y me $2.59 \quad 0.84 \quad 2.29 \quad 0.84 \quad 632.50 \quad 0.126 \quad 0.35$

mis estados emocionales. (8)

Tuve una experiencia negativa hacia el aprendizaje de técnicas de desar rollo personal y emocional, $1.68 \begin{array}{lllllll}0.76 & 1.55 & 0.69 & 709.00 & 0.450 & 0.18\end{array}$

bien por experiencias pasadas falta de interés o de motivación. (9)

\begin{tabular}{lllllllll}
\hline Conozco los principios básicos de la técnica del M indfulness. (10) & 2.61 & 0.89 & 2.00 & 0.93 & 496.50 & 0.004 & 0.67
\end{tabular}

\begin{tabular}{lllllllll}
\hline Practico el Mindfulness como técnica de desar rollo de las habilidades per sonales y emocionales. (11) & 2.29 & 0.87 & 1.63 & 0.78 & 45.2 & $<0.001$ & 0.79 \\
\hline
\end{tabular}

Dimensión desarrollo profesiona

En clase de EF planifico y llevo a cabo contenidos para desar rollar las habilidades emocionales y el $\begin{array}{llllllll}4.66 & 6.24 & 3.79 & 5.80 & 713.00 & 0.488 & 0.14\end{array}$ desarrollo personal. $(*)$

\begin{tabular}{llllllll}
\hline Aplico la práctica del Mindfulness en las clases de EF. $\left(^{*}\right)$ & 3.17 & 6.28 & 0.61 & 1.81 & 620.00 & 0.034 & 0.55 \\
\hline
\end{tabular}

\section{Dimensión dificultades de aplicación}

\begin{tabular}{llllllll}
\hline Mi disposición para impartir técnicas de desarrollo personal con el mindfulness en clase de EF es 1.73 & 0.59 & 1.97 & 0.85 & 673.50 & 0.256 & -0.33
\end{tabular} negativa. (17) \begin{tabular}{l}
\hline No practico técnicas de desar rollo personal en clase de EF debido a la mala disposición de los 1.90 \\
alumnos (falta de interés, apatía, etc.). (18)
\end{tabular}

\begin{tabular}{llllllll}
\hline No practico técnicas de desarrollo personal en clase de EF debido a la falta de tiempo para 2.12 & 0.84 & 2.39 & 0.92 & 647.00 & 0.173 & -0.31
\end{tabular} temporalizar y desar rollar este tipo de contenido. (19)

No practico técnicas de desarrollo personal en clase de EF debido a que no dispongo de los

materiales e instalaciones (equipo de música, colchonetas, tatami, acústica, temperatura de la $2.20 \quad 0.90 \quad 2.24 \quad 0.91 \quad 744.00 \quad 0.722 \quad-0.05$ instalación inadecuada, etc.). (20)

Fuente: elaboración propia. Nota: M (media), Mdn (mediana), DT (desviación típica), valor de U MannW hitney (U), valor significativo (p), valor d de

Cohen (d ), dimensión formación (1-5), dimensión experiencia per sonal (6-11), dimensión desar rollo profesional $\left({ }^{*}\right)$ y dimensión dificultades (17-20). Singularidad= Comunalidad.

Tabla 5.

Pesos de losítems y solución factorial provisional.

\begin{tabular}{|c|c|c|c|c|}
\hline & \multicolumn{3}{|c|}{ Factor } & \multirow[b]{2}{*}{ Singularidad } \\
\hline & 1 & 2 & 3 & \\
\hline $\begin{array}{l}\text { Item1. Formación específica sobre técnicas de desar rollo per sonal e } \\
\text { inteligencia emocional }\end{array}$ & 0.56 & & & 0.56 \\
\hline $\begin{array}{l}\text { Item2. Considero que, sal i de la carrera, total mente preparado para } \\
\text { desar rollar las competencias emocionales en mi futura labor como docente }\end{array}$ & 0.86 & & & 0.28 \\
\hline Item3. Profesores empleaban una metodología innovadora & 0.83 & & & 0.27 \\
\hline $\begin{array}{l}\text { Item4. Profesores prestaban más atención al desar rollo de las competencias } \\
\text { emocionales }\end{array}$ & 0.81 & & & 0.30 \\
\hline Item5. En la Universidad, existe una gran oferta & 0.63 & & & 0.63 \\
\hline $\begin{array}{l}\text { Item6. Tengo conocimientos y experiencia sobre las técnicas de desar rollo } \\
\text { personal y emocional. }\end{array}$ & 0.34 & 0.51 & -0.333 & 0.46 \\
\hline tem7. Mi disposición hacia la práctica es positiva & & 0.42 & & 0.75 \\
\hline Item8. Practico técnicas de desar rollo per sonal y emocional semanalmente. & & 0.74 & & 0.41 \\
\hline Item9. Tuve una experiencia negativa hacia el aprendizaje. & 0.39 & & 0.36 & 0.64 \\
\hline Item10. Conozco los principios básicos de la técnica del Mindfulness. & & 0.87 & & 0.27 \\
\hline Item11. Practico el Mindfulness & & 0.87 & & 0.27 \\
\hline Item12*. (17). Mi disposición para impartir técnicas es negativa. & 0.41 & & & 0.69 \\
\hline $\begin{array}{l}\text { Item13*. (18). No practico técnicas de desarrollo personal en cl ase de EF } \\
\text { debido a la mala disposición de los al umnos (falta de interés, apatía, etc.). }\end{array}$ & & & 0.55 & 0.61 \\
\hline $\begin{array}{l}\text { Item14*. (19). No practico técnicas de desarrollo personal en clase de EF } \\
\text { debido a la falta de tiempo para temporalizar. }\end{array}$ & & & 0.78 & 0.33 \\
\hline $\begin{array}{l}\text { Item15*. (20). No practico técnicas de desarrollo personal en clase de EF } \\
\text { debido a que no dispongo de los materiales e instal aciones. }\end{array}$ & & & 0.72 & 0.50 \\
\hline
\end{tabular}

Nota: Los ítems (17, 18, 19 y 20), tienen en el análisis los ítems con los valores $\left(12^{*}, 13^{*}, 14^{*}, 15^{*}\right)$

respectivamente. Singul aridad $=$ Comunalidad.

Tabla 6

Pesos de losítems y solución factorial definitiva.

\begin{tabular}{|c|c|c|c|c|}
\hline & \multicolumn{3}{|c|}{ Factor } & \multirow[b]{2}{*}{ Singularidad } \\
\hline & 1 & 2 & 3 & \\
\hline $\begin{array}{l}\text { Item1. Formación específica sobre técnicas de desar rollo per sonal e } \\
\text { inteligencia emocional. }\end{array}$ & 0.55 & & & 0.60 \\
\hline $\begin{array}{l}\text { Item2. Considero que, salí de la car rera, total mente preparado para } \\
\text { desar rollar las competencias emocionales en mi futura labor como docente. }\end{array}$ & 0.84 & & & 0.30 \\
\hline Item3. Profesores empleaban una metodología innovadora & 0.84 & & & 0.24 \\
\hline $\begin{array}{l}\text { Item4. Profesores prestaban más atención al desar rollo de las competencias } \\
\text { emocionales. }\end{array}$ & 0.82 & & & 0.30 \\
\hline Item5. En la Universidad, existe una gran oferta. & 0.65 & & & 0.60 \\
\hline Item8. Practico técnicas de desar rollo personal y emocional semanalmente. & & 0.68 & & 0.50 \\
\hline Item10. Conozco los principios básicos de la técnica del Mindfulness. & & 0.84 & & 0.30 \\
\hline Item11. Practico el Mindfulness & & 0.91 & & 0.18 \\
\hline $\begin{array}{l}\text { Item13*. (18). No practico técnicas de desarrollo per sonal en clase de EF } \\
\text { debido a la mala disposición de los alumnos (falta de interés, apatía, etc.). }\end{array}$ & & & 0.55 & 0.62 \\
\hline $\begin{array}{l}\text { Item } 14 * \text {. (19). No practico técnicas de desarrollo per sonal en clase de EF } \\
\text { debido a la fal ta de tiempo para temporalizar. }\end{array}$ & & & 0.76 & 0.35 \\
\hline $\begin{array}{l}\text { Item15*. (20). No practico técnicas de desarrollo per sonal en clase de EF } \\
\text { debido a que no dispongo de los materiales e instalaciones. }\end{array}$ & & & 0.75 & 0.46 \\
\hline
\end{tabular}

encontró ninguna diferen ción del plan de estudios para los ítems del cuestionario (todas las p's >0.05).

LaTabla 5 señala el análisis confirmatorio y exploratorio, se presentan todos los ítems originales del cuestionario. Trasel análisis exploratorio se eliminaron 4 ítems: los ítems 6 y 9 por tener más de 0.25 de peso en más de un factor y los ítems 7 y 12 por tener unas comunalidades inferiores a 0.35 (singularidades superiores a 0.65 ). Los 11 ítems restantes (Tabla 6) presentaron unos valores de adecuación de la muestra aceptables, mostrando un valor medio del índice KMO de 0.70 , con valores que iban desde 0.58 hasta 0.82 (Field, 2009). La prueba de Bartlett confirmó la esfericidad de la matriz $c^{2}(55)=412.37, p<$ 0.001. La solución final estabacompuestapor tres factores (Figura 1) que en conjunto explicaban el $59.75 \%$ de la varianza. El Factor 1 (Formación) estaba compuesto por los cinco primeros ítems y explicaba el $26.73 \%$ de la varianza. El Factor 210 formaban los ítems 8, 10 y 11 explicando el $19.12 \%$ de lavariabilidad, mientrasque el Factor 3 lo integraban los tres últimos factores, y explicaba el 13.90\%. La correlación encontrada entre factores fue baja: Factor 1 con el Factor $2(r=0.13)$ y el Factor $3(r=0.27)$; Factor 2 y $3(r=-0.07)$. La Tabla 6 muestra los 


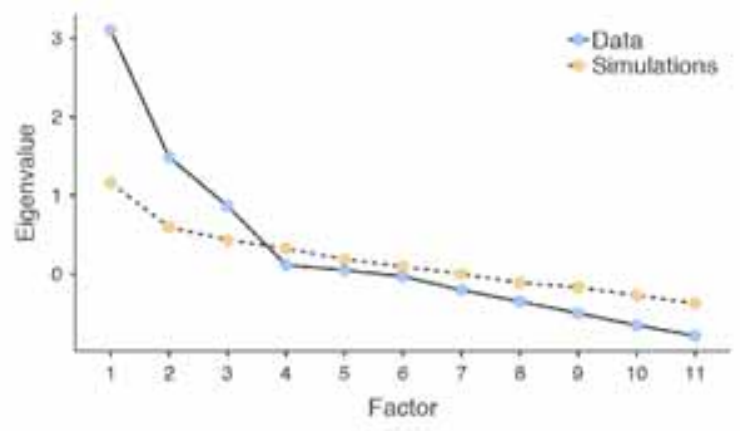

Figura 1. Gráfico de sedimentación. Datos (---); sedimentación (---).

nuestro planteamiento fue conocer los factores que pueden producir esta problemática.

En relación con los resultados obtenidos y siguiendo las aportaciones de la literatura, podemos afirmar según Joyce et al. (2010), que la importancia de la forma ción de los docentes respecto a la adquisición de técnicas de desarrollo en habilidades de la IE en la universidad, tiene relativa importancia para poder cooperar a acrecentar, los futuros procesos de enseñanza aprendizaje en el aula. El objetivo principal es, que los docentes

Tabla 7

Análisis de ítems y análisis de consistencia interna

\begin{tabular}{|c|c|}
\hline Variables dimensiones & $\begin{array}{l}\text { Correlación a Cronbach } \\
\text { ítem- factor si se elimina el ítem }\end{array}$ \\
\hline \multicolumn{2}{|l|}{ DIMENSIÓN FORMACIÓN Alfa de Cronbach 0.84} \\
\hline He recibido for mación específica sobre técnicas de desar rollo per sonal e inteligencia emocional.(1) & 0.86 \\
\hline $\begin{array}{l}\text { Considero que salí de la car rera, totalmente preparado para desarrollar las competencias emocionales en mi futura } \\
\text { labor como docente.(2) }\end{array}$ & 0.74 \\
\hline $\begin{array}{l}\text { Los profesores que tuve en la Univer sidad, empleaban una metodología innovadora para desar rollar las habilidades en } \\
\text { inteligencia emocional en sus clases, de esta for ma pude adquirir técnicas de forma indirecta. (3) }\end{array}$ & 0.71 \\
\hline $\begin{array}{l}\text { Los profesores prestaban más atención al desar rollo de las competencias emocionales inter per sonales e intraper sonal } \\
\text { que a la teoría y la práctica deportiva. (4) }\end{array}$ & 0.75 \\
\hline $\begin{array}{l}\text { En la Univer sidad, existe una gran oferta para poder formar me en este ámbito y poder incorporarlo en mi profesión } \\
\text { como docente de educación física. (5) }\end{array}$ & 0.60 \\
\hline \multicolumn{2}{|l|}{ DIMENSION EXPERIENCIA PERSONAL Alfa de Cronbach 0.85} \\
\hline $\begin{array}{l}\text { Practico técnicas de desar rollo per sonal y emocional semanal mente, me proporciona bienestar y me ayuda a } \\
\text { autorregular mis estados emocionales. (8) }\end{array}$ & 0.64 \\
\hline Conozco los principios básicos de la técnica del Mindfulness. (10) & 0.76 \\
\hline Practico el Mindful ness como técnica de desar rollo de las habilidades per sonales y emocionales. (11) & 0.74 \\
\hline \multicolumn{2}{|l|}{ DIMENSIÓN DIFICULTADES DE APLICACIÓN Alfa de Cronbach 0.73} \\
\hline $\begin{array}{l}\text { No practico técnicas de desarrollo per sonal en clase de EF debido a la mala disposición de los alumnos (falta de } \\
\text { interés, apatía, etc.). (13*) (18) }\end{array}$ & 0.49 \\
\hline $\begin{array}{l}\text { No practico técnicas de desarrollo per sonal en clase de EF debido a la falta de tiempo para temporalizar y desar rollar } \\
\text { este tipo de contenido. }\left(14^{*}\right)(19) \text {. }\end{array}$ & 0.62 \\
\hline $\begin{array}{l}\text { No practico técnicas de desarrollo personal en clase de EF debido a que no dispongo de los materiales e instalaciones } \\
\text { (equipo de música, colchonetas, tatami, acústica, temperatura de la instalación inadecuada, etc.). (15*) (20). }\end{array}$ & 0.58 \\
\hline
\end{tabular}

pesos de los ítems y solución factorial definitiva.

En la Tabla 7 señala los resultados del análisis de la fiabilidad. Se observó que la subescala de Formación (Factor 1) mostró un coeficientealfade 0.84 , lasubescala de Práctica (Factor 2) de 0.85, mientras que la subescala de Barreras (Factor 3) presentó un coeficiente de 0.73. En cuanto a la escala global, en resumen, todas ellas estuvieron por encima de 0.70 , lo que indica una consistencia aceptable (Tavakol \& Dennick, 2011).

\section{Discusión}

El presente estudio se planteó para conocer en las clases de EF de secundaria, el estado de la práctica de las técnicas de desarrollo de la IE y en especial la de mindfulness, así como las posibles dificultadesen lapráctica. Para ello, se recabó la información de 79 profesores en cuatro apartados principales como son su formación inicial sobe el tema, la práctica personal, la práctica profesional y las barreras percibidas para su inclusión en las clases. En este sentido, los resultados confirman la presencia de dificultades en la inclusión de técnicas en habilidades de desarrollo de la IE y mindfulness en el curriculum educativo. Partiendo de esta objetividad, sientan que han salido formados de la universidad para desarrollar las competencias emocionales en el área de EF. Este hecho tan relevante en la educación, favorece tanto a los docentes como a los alumnos en mejorar el nivel de inteligencia emocional y así poder optimizar su capacidad cognitiva y de atención, para ser más conscientes de su entorno y de sí mismos.

Bueno, Teruel \& Valero (2005), tal y como señalan en su estudio empírico, abordan el manejo de algunas dimensiones de la IE y exposición, frente a una situación problemática dentro del contexto educativo de los futuros maestros de varias especialidades del primer y tercer curso de Magisterio. El estudio confirmó que existe una falta de habilidad de gestión de las emociones respecto a la claridad emocional, atención de las emociones y reparación de las mismas de los futuros docentes. Por consiguiente, se reafirma con los resultados obtenidos en el presente estudio realizado, que los docentes consideran que no han salido preparados para desarrollar las competencias emocionales en su especialidad.

Una de las conclusiones obtenidas en el presente estudio, es la afirmación respecto a que una de las principales causas que dificultan la inclusión de las competencias emocionales en área de EF, se debe a la falta de conocimiento del mindfulness por parte de los docentes. Este hecho, dificulta su práctica a nivel profesional como a nivel personal, repercutiendo en descartar la planificación de sesiones de mindfulness en el área de EF. Joyce et al. (2010) sugieren para mejorar la forma ción de los docentes, que antes de llevar a la práctica del 
programa de mindfulness por parte del profesorado al aula, primeramente adquieran una preparación en su desarrollo personal, se pretende por tanto, que puedan experimentar, identificar y gestionar de la forma más adecuada las emociones y poder trasladar todo este tra bajo de desarrollo personal y emocional a los alumnos en el aula. Se necesita de una preparación y conocimiento para su posterior enseñanza en el aula que según afirma Santorelli (2014), la creciente expansión de las técnicas del mindfulness, viene afiliado a un amplio número de personas sin la formación adecuada.

Según Joyce et al. (2010), en su estudio observaron quelos docentes comprobaron al gunas dificultades cuando iban a poner en marcha el programa de mindfulness en el aula, uno de los mayores obstáculos fue, la falta de tiempo para implementar el programa. 0 tro factor, es la falta de recursos formativos por parte del profesor para afrontar situaciones de estrés y hacer frente a estudiantes que no querían involucrarse y participar en el programa. En relación con lo mencionado, en el presente estudio tras anal izar el factor barrera, denomina da: dificultades de aplicación del mindful ness como técnica de habilidades de desarrollo de la IE en el aula, se observó una diferencia en los resultados respecto al estudio realizado por el grupo de investigación de Joyce et al. (2010), Arthurson (2015) y Desmond (2010).

Por un lado, Arthurson (2015) en su investigación, considera como dificultades de aplicación por parte de los docentes para este tipo de práctica en los centros educativos, la fal ta o escasa disposición tanto de materiales como de instalaciones apropiadas. Por otro lado, Desmond (2010) afianzan que un factor que imposibilita la inclusión de estas técnicas en el aula, es debido al cumplimiento de la programación y tareas administra tivas, en consecuencia al tiempo que se dispone. En este estudio se observó, que a pesar de que los profesores no recibieron la formación en este ámbito en la universidad, ni admitían salir preparados, muestran un interés positivo en el conocimiento de estas técnicas para poder abordar personal y profesionalmente las competencias emocionales, sin tener en el ámbito de desarrollo personal ningunaexperiencianegativa. Losdocentesque se formaron y que incluyeron en la programación anual al guna técnica en desarrollo de la habilidad emocional y personal o de mindfulness con los alumnos, reflejaron que la falta de tiempo, de instalaciones y de material, no fueron factores barrera. Igualmente, pudieron apreciar los beneficios en los alumnos derivados de técnicas de desarrollo de habilidades emocionales.

Shapiro, Warren \&Astin (2008), afirman que la prác- tica de alguna técnica de desarrollo de IE al aula, hace que el al umno experimente una serie de beneficios: una mayor calma interna, aumenta la capacidad de autocontrol y de autoestima, adquiere una mayor habilidad para identificar su emociones y de gestionarlas, aumenta la interacción social y la capacidad de concentración favoreciendo el nivel académico.

0 tro dato relevante, es que se obtuvieron resulta dos diferentes en cuanto a la disposición de los alumnos respecto al estudio realizado por Joyce et al. (2010). Mencionar que el grupo minoritario de profesores que pusieron en práctica al guna técnica de desarrollo de IE, afirmaron que los alumnos que participaron, no registraron una disposición negativa hacia la práctica de técnicas de desarrollo personal. Joyce et al. (2010), sujetan como resultado en uno de sus estudios que los profesores relataban que no tenían recursos para hacer frente a los alumnos que no querían participar en la actividad, y no participaban de forma adecuada en el programa. La escasez de una apropiada predisposición y formación por parte del docente que realizalastécnicas del mindfulness, podría acotar o dificultar la obtención del propósito (Delgado, 2009).

No se han encontrado estudios que en la que poda mos comparar los resultados hallados en nuestra investigación, en cuanto a las diferencias encontradas entre las variables de sexo y plan de estudios impartiendo clases de EF de los docentes. Por lo que las diferencias por sexo podrían deberse a que las mujeres, al estar más predispuestas y positivas hacia la práctica de técnicas de desarrollo personal y emocional, se han involucrado en su formación y aprendizaje. Cabe desta car que existen diferencias de género en las técnicas de regulación de la emoción, así lo demuestran las investigaciones por neuroimagen informando que los hombres demuestran una menor activación de las regiones del cerebro involucradas en la regulación emocional (regiones prefrontales asociadas con la regulación de la emoción, amígdala y regiones asociadas a la recompensa) (M cRae et al., 2008). M ientras que las mujeres demuestran un efecto negativo disminuido y una mayor capacidad de atención y autocompasión. Los tratamientos específicos de género pueden ser más destacados para los hombres y requerir intervenciones de atención plena que se ajusten a los estilos de afrontamiento particulares que tienden a usar (Rahil et al., 2017).

También cabe mencionar en cuanto a las relaciones encontradas entre los diferentes ítems de las dimensiones formación, experiencia personal y dificultades de aplicación con el $n$ no de sesiones de práctica en clase de 
EF en habilidades emocional es y de mindfulness. Se observó una correlación positiva entre los profesores que recibieron formación y el $n-$ se sesiones de habilidades emocionales, no existiendo una correlación positiva con el $n$ - de sesiones de mindfulness realizadas en clase de EF. Este hecho, puede estar asociado a la falta específica de formación sobre mindfulness y su dominio (Delgado, 2009). Se observó una correlación negativa en cuanto al $n$ - de sesiones de habilidades realizadas en clase de EF y el ítem que los profesores no practicaban las técnicas de desarrollo personal en clase de EF debido a la falta de tiempo, instalaciones, material, etc. Basándonos en las aportaciones de Delgado (2009), el dominio y la buena planificación facilita el desarrollo de la práctica en el aula.

Por otro lado, en la dimensión experiencia personal en técnicas de desarrollo personal y emocional, se observó que los docentes que experimentaron un aprendizaje voluntario hacia el conocimiento de las técnicas, presentan una correlación muy positiva en cuanto a los ítems (conocimiento y experiencia de técnicas de desarrollo personal, una disposición positiva hacia la práctica, la práctica de las técnicas de desarrollo personal semanalmente, conocimiento de los principios básicos de la técnica del mindfulness y por consiguiente su práctica personal) y la práctica en $\mathrm{n}$ - de sesiones desarrolladas en clase de EF. Además, cabe destacar que los docentes que si dominaban la técnica de mindfulness y la forma de implementarlo en el aula, presentan una correlación más alta en cuanto al no de sesiones en mindfulness rea lizadas en clase, respecto alos docenes que conocen otras técnicas (Greenland, 2010).

En conclusión, los resultados de esta investigación muestran que la falta de formación recibida en la universidad y el desconocimiento del mindfulness como método y sus beneficios, son las principales dificultades para incluir sesiones de habilidades y técnicas como el mindfulness para el desarrollo de la IE en clase de EF. Lo que sugiere una mayor concienciación de los propios docentes universitarios en la necesidad de formar a sus futuros maestros para responder a las necesidades y abordar las competencias emocionales del currículum en el área de EF, como potenciador a la mejora de la salud física y psicológica, de la capacidad atencional y fomento del bienestar personal.

\section{Referencias}

Acebes-Sánchez, J., \& Rodríguez-Romo, G. (2019). Relaciones entre el nivel de actividad física, expe- riencia deportiva e inteligencia emocional en alumnos de Grado de la Comunidad Madrid: Estudio de protocolo. Retos, 36, 297-301.

Arthurson, K. (2015). Teaching mindfulness to year sevens as part of health and personal development. Australian Journal of Teacher Education (Online), 40(5), 27. Recuperado de https:/ / ro. ecu. edu. au/ cgi/ view content. cgi?article $=2605 \&$ context $=$ ajte

Association Mindful Schools (2007). Courses. Recuperado de https:/ / www. mindfulschools.org

Berrocal, P., \& Extremara, N. (2002). La inteligencia emocional como una habilidad esencial en la escuela. Revista Iberoamericana Educativa, 29, 1-6. Recuperado de https:/ / www. researchgate. net/ publication / 230886932 La_inteligencia_emocional_como _una_habilidad_esencial_en_la_escuela

Berrocal, P. , \& Ruiz, D. (2008). La İnteligencia emocional en laEducación. Revista Electrónica de Investigación Psicoeducativa, 2, 421-436. Recuperado dewww. academia. edu/ 4695295/ Fern\%C3\%Alndez

Berrocal_P._Extremera_N._y_Ramos_N. 2003_._Inteligencia_emocional_y_depresi\%C3\% B33n. Encuentros_en_Psicolog\%C3\%ADa_Social_1 51254

Bisquerra, R. (2008). Educación para la ciudadanía. El enfoque de la educación emocional. Barcelona: Wolters Kluwer.

BOCM (2015). DECRETO 48/ 2015, de 14 de mayo, del Consejo de Gobierno, por el que se establece para la Comunidad de Madrid el currículo de la Educación Secundaria Obligatoria. Recuperado de http:/ / www.bocm.es/ Bueno, Ma , Teruel, C., \& Valero, A. (2005). La inteligencia emocional en alumnos de M agisterio: la percepción y comprensión de los sentimientosy lasemociones. Revista interuniversitaria de Formación del Profesorado, 19 (3), 169-194. Recuperado de file:/ / C:/ U sers/ U ser/ Desktop/ MÁSTER\%20CCAFD/ TFM \% $20 \mathrm{M}, \mathrm{F}, \% 20 \mathrm{SA}$ N D R A / D ial netLalnteligenciaEmocionalEnAlumnosD eM agisterio2126774\%20(1).pdf

Canales, I., \& Rovira, G. (2017). La práctica de la atención plena en estudiantes universitarios. Dificulta des y facilidades percibidas. Retos, 31, 118-122.

Cardaciotto, A. (2005). Assessing mindfulness: The development of a bi-dimensional measure of awareness and acceptance. Tesis D octoral no publicada presentada en la Universidad de Drexel.

Cohen, J. (1988). Statistical power analysisfor the behavioural science (2nd ed.). New York:Academic Press.

Constantino, S., \& Espada, M.(2021). Análisis de los 
canales de desarrollo e inteligencia emocional mediante una intervención de una unidad didáctica de M indfulness y Biodanza en Educación Física para Secundaria. Retos, 40, 67-75.

Delgado, J. C. (2009). Correlatos psicofisiológicos de Mindfulness y la preocupación, Eficacia de un entrenamiento en habilidades mindful ness. Tesis doctoral. Editorial de la U niversidad de Granada.

Desmond, C. T., \& Hanich, L. (2010). The effects of mindful awareness teaching practices on the executive functions of students in an urban, low income middle School. Millersville U niversity: Pennsylvania Research Report 1(12). Recuperado de https:/ / pdfs.semanticscholar.org/ 47 a0 / e89e2 e6fb8341b867ca7cfob45fc349d6371. pdf

Dickenson, J., Berkman, E., Arch, J., \& Lieberman, M. (2013). N eural correlates of focused attention during a brief mindfulness induction. Revista de Social Cognitive and Affective N euroscience, 8.40-47. Recuperado de https:/ / academic. oup.com/ scan/ article/ 8/ 1/ 40/ 1694064

Espada, M. , \& Calero, J. C. (2012). La inteligencia emocional en el área de Educación Física. La Peonza: Re vista deEducación Física para la paz, 7, 65-69. Recuperado de https: / / dialnet. unirioja. es/ servlet/ articulo?codigo $=3907255$

Fernández, G., \& N avarro, V. (1989). Diseño curricular en Educación Física. Barcelona: Inde.

Field, A. (2009). D iscovering statistics using SPSS ( $3^{2}$ ed. ). London: Sage.

Franco, C., \& M añas, I. (2010). Reducción de los niveles de estrés docente y de los días de baja laboral por enfermedad en profesores de educación secundaria obligatoriaatravés de un programa de entrenamiento en meditación. En prensa.

Franco, C. , Mañas, I., \& Justo, E. (2009). Reducción de los niveles de estrés, ansiedad y depresión en docentes de educación especial a través de un programa de mindfulness. Revista Educación inclusiva, 2(3), 1133. Recuperado de http:/ / www. ujaen.es/ revista/ rei/ linked/ documentos/ documentos/4-1. pdf

González, A. (2013). Mindfulness (atención plena o conciencia plena). Guía para educadores. Recuperado de file:/ / / C:/ U sers/ usuario/ Downloads/ MINDFULNESS al aznegonzalez.pdf

Greenland, S.K. (2010). The mindful child: How to help your kid manage stress and become happier, kinder, and more compassionate. Simon and Schuster.

Hair, J., Anderson, R., Black, B. \& Babin, B. (2016). Multivariate Data Analysis (7th ed.): Pearson
Education.

Hölzel, B. , Carmodyc, J., Vangela, M., Congletona, C., Yerramsettia, S., Garda, T., \& Lazara, S. (2011). Mindfulness practice leads to increases in regional brain gray matter density. Revista National Institutes of Health, 191(1), 36-43. Recuperado de https:// ww w. ncbi.nlm.nih.gov/ pmc/ articles/ PMC3004979/

Horn, J. L. (1965). A rationale and test for the number of factors in factor analysis. Psychometrika, 30, 179185. doi: 10.1007/ BF02289447

Joyce, A., Etty-Leal, J., Zazryn, T., \&H amilton, A. (2010). Exploring a mindful ness meditation program on the mental health of upper primary children:A pilot study. Advances in School Mental Health Promotion, 3(2), 17-25.

Kabat-Zinn, J. (1990). Full Catastrophe Living. N ew York: Delta.

Kabat-Zinn, J. (2010). El poder de la atención: 100 lecciones sobre el Mindfulness. Barcelona: Kairós.

León, B. (2008). Atención plena y rendimiento académico en estudiantes de enseñanza Secundaria. European Journal of Education and Psychology, 1(3), 1726. Recuperado de https: / dial net. unirioja. es/ servlet/ articulo?codigo=2761660file: / / C:/ U sers/ U ser/ AppData/ Local/ Packages/ Microsoft. M icrosoftEdge_8weky b3d8bbwe/ TempState/ Downloads/ 523-2025-1-PB.pdf

Leung, M. K., Chan, C., Yin, J., Lee, C., Fai So, K., \& Lee, T. (2013).Increased gray matter volume in the right angular and posterior parahippocampal gyri in loving-kindness meditators. Revista de Social Cognitive and Affective Neuroscience, 8(1), 34-39. R ecuperado de https:/ / academic. oup.com/ scan/ article/ 8/ 1/ 34/ 1696981

López, L. (2013). Programa «TREVA» (Técnicas de Relajación Vivencial aplicadas al Aula: A plicaciones. eficacia y acciones formativas). ISEP, 4, 26-32.

López, L.,Amutio, A., O riol, X., \& Bisquerra, R. (2016). Mindfulness e investigación acción en educación secundaria. Gestación del programa TREVA. Revista Interuniversitaria de formación del profesorado, 87 (30), 75-91. Recuperado de https:/ / dialnet.unirioja.es/ servlet $/$ articulo?codigo $=5802514$

Lu, C. , Tito. J., \& Kentel, J. (2009). Eastern movement disciplines (EM Ds) and mindfulness:A new path to subjective knowledge inWestern physical education, 61(3), 353-370.

Luders, E., Kurth, F., Mayer, E., Toga, A., Narr, K., $\&$ Gaser, C. (2012). The U nique Brain Anatomy of Meditation Practitioners: Alterations in Cortical Gyrification. Front H um N euroscience, 6(34). Recupe- 
rado de https: / / www. ncbi.nlm.nih.gov/ pmc/ articles/ PM C3289949/

Mañas, I., Franco, C., Gil, M a , \& Gil, C. (2014). Educación consciente: mindfulness en el ámbito educativo. Educadores conscientes formando a seres humanos conscientes. Alianza de civilizaciones, políticas migratorias y educación. Sevilla: Aconcagua Libros.

Matthieu, R., Lutz, A., \& Davidson, R. (2015). En el cerebro del medidor. Revista Investigación y Ciencia, 460(18-25). Recuperado de https: / / www. investig acionyciencia.es/ revistas/ investigacion-y-ciencia/ neurociencia-de-la-meditacin-618/ en-el-cerebrodel-meditador-12725

Mayer, J. D., \& Salovey, P. (1997). W hat is emotional intelligence? En P. Salovey y D. Sluyter (Eds). Emotional Development and Emotional Intelligence: Implications for Educators (pp. 3-31). N ew York: Basic Books. Recuperado de http:// ei.yale.edu/ w pcontent/ uploads/ $2014 / 02$ / pub219_Mayer_Salovey_1997.pdf

McRae, K., O chsner, K., M auss, I., Gabrieli, J., \& Gross, J. (2008). Gender Differences inEmotion Regula tion: An fMRI Study of Cognitive Reappraisal. Sage Journal, 11(2). Recuperado de https:/ / doi.org/ $10.1177 / 1368430207088035$

Mindful Schools. (2007). Emeryville. 0 akland. CA. Recuperado de https:/ / www. mindfulschools.org/ about-mindfulness/ our-organization/ ?br=ro

Modrego, M., Martínez, L., López, A., Borao, L., Margolles, R., \& Campayo, J. (2016). Mindfulness en contextos educativos: profesores que aprenden y profesores que enseñan mindfulness. Revista Interuniversitaria de formación del profesorado, 87(30.3), 31-46. Recuperado de https:// javiergar ciacampayo4. files. wordpress.com/ 2016/ 12/ articc81culo-revista-profesores-que-aprenden-y-profesores-que-ensencc83an. pdf

Monfort, M. (2015). La creatividad en la expresión corporal. Un estudio de casos en educación secundaria. Educación Física y Deportes, 122(4), 28-35. Recuperado de http:/ / www. revista-apunts.com/ es/ hemeroteca?article $=1708$

OCM (2015). DECRETO 48/ 2015, de14 demayo del Consejo de Gobierno por el que se establece para la Comunidad de Madrid el currículo de la Educación Secundaria Obligatoria. Recuperado de http:/ / www.bocm.es/

Poulín, P.A. (2009). M indfulness-based wellness education: A longitudinal evaluation with students in initial teacher education. Unpublished doctoral dissertation. University of Toronto. Canadá. Recuperado de https: / / tspace.library. utoronto.ca/ bitstream/ $1807 / 26538 / 1 /$ Poulin_Patricia_A_200911_ $\mathrm{PhD}$ thesis.pdf

Ragoonaden, K. (2016). SmartEducation: Developing Stress M anagement and Resiliency Techniques. Recuperado de https: / / www. learninglandscapes.cal index. php/ learnland/ article/ view/ smartEducation-D eveloping-Stress- $M$ anagement-andResiliency-Techniques/ 815

Rahil, R., Santoyo, J., Rahrig, H., Roth, H., \& Britton, W. (2017). Women Benefit More Than Men in Response to College-based Meditation Training. Frontiers in Psychology. Recuperado de https:/ / ww w. frontiersin.org/ articles/ 10.3389/ fpsyg 2017.00551/ full

Riera, J. (1985). Introducción a la psicología del deporte. Barcelona: M artínez.

Ros, A. (2013). Inteligencia emocional y deporte: situación actual del estado de la investigación. Cuadernos de Psicología del Deporte, 13(1), 105-112. Recuperado dehttp:/ / scielo. isciii. es/ pdf/ cpd/ v13n1/ art10. pdf Santorelli, S. (2014). M indfulness-Based Stress Reduction (MBSR). Standards of Practice Background and $O$ verview. University of $M$ assachusetts Medical School: Center for Mindfulness in Medicine, Health Care \& Society.

Shapiro, S. , Warren, K., \& Asti, J. (2008). Toward the Integration of Meditation into Higher Education. A Review of Research. Recuperado de http:/ / w w w. contemplativemind.org/ files/ MedandHigherEd.pdf

Siegel, D. J. (2007). TheM indful Brain. N ewYork: NortonCompany.

Tavakol, M.,\& Dennick, R. (2011). Making sense of Cronbach's alpha. International journal of medical education, 2, 53-55. doi: 10.5116/ ijme. 4dfb. 8dfd

Trujillo, D., \& Reyes, M. (2019). Programa basado en mindfulness para la reducción de la ansiedad precompetitiva en deportistas de artes marciales. Retos, 36, 418-426.

Worthington, R. L., \& W hittaker, T. A. (2006). Scale development research: A content analysis and recom mendations for best practices. The Counseling Psychologist, 34, 806-838. 